\title{
REFLECTION GROUPS AND A CONVOLUTION THEOREM
}

\author{
Suren L. FERnANDo
}

The purpose of this paper is to prove a converse to a theorem of Eaton and Perlman on convolutions of $G$-decreasing functions. Both their result and our converse concern a connection between the theory of reflection groups and a class of probability inequalities that are of interest to statisticians. The original theorem states that the Convolution Theorem is satisfied by reflection subgroups of the orthogonal group. We show in this paper that if $G$ is a finite linear group that satisfies the Convolution Theorem, then $G$ is a reflection group. Furthermore, we show that if $\rho: G \hookrightarrow G L(V)$ is a faithful representation that satisfies the Convolution Theorem, then $\rho$ is a direct sum of the canonical Coxeter representation of $G$ and a trivial representation.

\section{Introduction.}

Let $V$ be a finite dimensional real vector space, and let $G \hookrightarrow G L(V)$ be a linear group. A function $f: V \rightarrow \mathbf{R}$ is said be $G$-decreasing provided that $f(x) \geq f(y)$ whenever $x$ is contained in the convex hull of the $G$-orbit of $y$. In [EP1], Eaton and Perlman showed that if $V$ is a Euclidean space and $G$ is the closure of a reflection subgroup (i.e., a subgroup generated by reflections) of the orthogonal group $O(V)$, then the convolution of a pair of $G$-decreasing real valued functions defined on $V$ is itself a $G$-decreasing function. For this reason such groups are said to satisfy the Convolution Theorem.

Complementing their convolution theorem, Eaton and Perlman showed in [EP2] that if $G$ is a closure of an infinite reflection subgroup of $O(V)$ that acts irreducibly on $V$, then $G=O(V)$. Using this result they deduced in [EP1] that if $G \subset O(V)$ is the closure of a reflection subgroup of $O(V)$, then $G$ can be decomposed into a direct product of a finite number of compact orthogonal groups and a finite reflection group; this decomposition also follows from the result on irreducible reflection groups cited above and the complete reducibility of representations of compact groups. Since the factors in the decomposition are reflection groups, the convolution theorem in [EP1] can be paraphrased as follows: The Convolution Theorem is satisfied by closed reflection subgroups of the orthogonal group $O(V)$. 
The results of Eaton and Perlman identify a class of much-studied groups that satisfy the Convolution Theorem. On the other side of the ledger, it has been observed by Eaton in Section 4 of [E2] that if $G$ is a finite cyclic group of order three or more, and $\rho: G \hookrightarrow G L\left(\mathbf{R}^{2}\right)$ is the rotation representation, then the Convolution Theorem does not hold for $G$. Since every finite subgroup of $G L\left(\mathbf{R}^{2}\right)$ is either a cyclic rotation group or a dihedral reflection group, the preceding observation of Eaton can be recast as follows: If $\operatorname{dim}_{\mathbf{R}} V=2$, then every finite group $G \subset G L(V)$ that satisfies the Convolution Theorem is a reflection group ${ }^{1}$. In Proposition 4.10, we show more generally that if $G$ is any finite linear group that satisfies the Convolution Theorem, then $G$ is necessarily a reflection group. This proposition and the main theorem of [EP1] together yield our main result, Theorem 4.11, which states that

\section{a finite linear group satisfies the Convolution}

Theorem if and only if it is a reflection group.

We also show that if the Convolution Theorem is satisfied for a faithful representation $\rho: G \hookrightarrow G L(V)$, then $\rho$ decomposes into a direct sum of the canonical Coxeter representation of $G$ and a trivial representation.

Theorem 4.11 determines all finite linear groups that satisfy the Convolution Theorem. Suppose, on the other hand, that $G \subset O(V)$ is an infinite group. If $G=O(V)$, then it is easy to see, and has been noted above, that $G$ satisfies the Convolution Theorem. In Section 4 of [E2] Eaton has observed that for a compact group $G$ to satisfy the Convolution Theorem it is adequate to require that $G$ acts transitively on a sphere in $V$. Since the special orthogonal group $S O(V) \subset O(V)$ is such a group that is not a reflection group, we see that Theorem 4.11 is not true in general for subgroups of $O(V)$, if the finiteness hypothesis is replaced by compactness (note that since $O(V)$ is compact, being closed and being compact are equivalent). A complete list of Lie groups that act effectively and transitively on spheres is known by the work of Montgomery and Samelson [MS] and Borel [Bor1], [Bor2]. In the cases where the dimension of $V$ is either even or 7 , there are compact Lie groups other than $S O(V)$ that act transitively and effectively on spheres in $V$.

While the applications of convolution theorems that the author is aware of are in mathematical statistics, the ideas involved in this paper derive primarily from the theory of Coxeter groups. In light of the mixed heritage of our topic, we have chosen to include some expository material on majoriza-

\footnotetext{
${ }^{1}$ Here, and in the rest of this paper, we consider the trivial group to be a reflection group with an empty set of generating reflections.
} 
tion, convex polytopes, and Coxeter groups. It is our hope that this will make the paper accessible to a wider audience.

We close this introduction by giving a brief outline of the contents of this paper. In Section 1 we discuss the notion of majorization and state the theorem of Eaton and Perlman cited above. In Section 2 we introduce the necessary background on convex polytopes, and in Section 3 we discuss Coxeter groups. Section 4 is devoted to a proof of our main result that a finite group satisfies the Convolution Theorem if and only if it is a Coxeter group.

\section{Majorization.}

The notion of majorization has its origins in the work of Muirhead [Mui] and Grace and Young [GraY]. The connection with multivariate statistics can be traced back to the 1955 paper [An] of Anderson, and extensions of Anderson's work by Sherman [Sh] and Mudholkar [Mud]; for a discussion of the influence of Anderson's seminal paper and a survey of subsequent work in multivariate statistics, see the commentary by Perlman $[\mathbf{P}]$. The concept of majorization is also implicit in the statement of the "Theorem of the Highest Weight" in the representation theory of compact Lie groups (see Chapter 6 of the monograph [Ad] by Adams for an explicit use of majorization in the statement of that result). The books by Hardy, Littlewood and Polya [HarLP], Marshall and Olkin [MaO1], Tong [To], and Eaton [E1] may be consulted for additional information on majorization. For a briefer introduction see the review by Kemperman [Ke] or Section 12 of Atiyah and Bott $[\mathrm{AtB}]$, which contains an elegant summary of the Lie theoretic aspects of the subject.

Our treatment of majorization will be phrased in representation theoretic terms. In what follows it will be assumed without further comment that all representations under consideration are finite dimensional real representations. By a linear group we shall mean an embedding (i.e., a faithful representation) $\rho: G \hookrightarrow G L(V)$ of a group $G$; although a linear group can be viewed as a subgroup of $G L(V)$, we shall often find it useful to make a distinction between the abstract group $G$ and the image of $G$ under $\rho$.

Let $\rho: G \hookrightarrow G L(V)$ be a linear group, and if $g \in G$, let $v \mapsto g \cdot v$ denote the action of $\rho(g)$ on $V$. Then a subset $A$ of $V$ will be said to be $\rho$-convex if the convex hull of the orbit of any element of $A$ under the action of $G$ is contained in $A$; that is, if

$$
t a+(1-t) g \cdot a \in A, \quad \text { whenever } a \in A, g \in G \text {, and } 0 \leq t \leq 1 .
$$

The $\rho$-convex hull, $\operatorname{conv}_{\rho}(A)$, of a set $A \subset V$ is the smallest $\rho$-convex subset of $V$ that contains $A$. Now define an "ordering" $\prec_{\rho}$ on $V$ by setting $x \prec_{\rho} y$ 
if

$$
x \in \operatorname{conv}_{\rho} y .
$$

If $x \prec_{\rho} y$, then $y$ will be said to majorize $x$ with respect to $\rho$.

An early occurence of majorization was in a result of Schur [Schu] according to which the diagonal elements of an $n \times n$ Hermitian matrix are contained in the convex hull of the orbit of the eigenvalues of the matrix, under the permutation action of the symmetric group $S_{n}$. If $x=\left(x_{1}, \ldots, x_{n}\right)$ are the diagonal entries of the matrix, $y=\left(y_{1}, \ldots, y_{n}\right)$ are the eigenvalues of the matrix, and $\rho: S_{n} \rightarrow G L\left(\mathbf{R}^{n}\right)$ is the permutation representation of $S_{n}$, then Schur's result asserts that $x \prec_{\rho} y$. In [Ho], Horn proved the converse result that if $x \prec_{\rho} y$, then there is an $n \times n$ Hermitian matrix with diagonal entries $\left(x_{1}, \ldots, x_{n}\right)$ and eigenvalues $\left(y_{1}, \ldots, y_{n}\right)$. This equivalence was extended to a Lie theoretic context by Kostant [Kos]. In turn, a part of Kostant's work was extended independently by Atiyah [At1] and GuilleminSternberg $[\mathbf{G u S}]$ to the setting of moment maps that arise from the action of a torus on a symplectic manifold.

If $\rho: G \hookrightarrow G L(V)$ is a linear group, then a function $f: V \rightarrow \mathbf{R}$ will be said to be $\rho$-decreasing if

$$
x \prec_{\rho} y \text { implies that } f(x) \geq f(y) \text {. }
$$

Following the definition in Eaton [E2], we shall say that the Convolution Theorem holds for $\rho$ if, for any two compact $\rho$-convex sets $A$ and $B$, the convolution

$$
\chi_{A} * \chi_{B}(x)=\int_{V} \chi_{A}(x-t) \chi_{B}(t) d t
$$

(with respect to Lebesgue measure on $V$ ) of their indicator functions is $\rho$ decreasing. As Eaton observes, if the Convolution Theorem holds, then the convolution of any two non-negative functions that are $\rho$-decreasing is itself $\rho$-decreasing, provided the convolution exists. In their 1974 paper [MaO2] Marshall and Olkin showed that the natural representation $\rho: S_{n} \rightarrow G L(n)$ of the permutation group $S_{n}$ on $n$ letters satisfies the Convolution Theorem. A few years later the result of Marshall and Olkin was extended by Eaton and Perlman [EP1] to all reflection groups that are closed subgroups of an orthogonal group $O(V)$, where $V$ is a Euclidean space. They showed that

$$
\text { the natural reflection representation of any closed }
$$$$
\text { reflection group satisfies the Convolution Theorem. }
$$

As mentioned above, the purpose of this paper is to prove a converse to this result, under the assumption that the group in question is finite. 


\section{Convex polytopes.}

This section will be devoted to a discussion of the necessary background from polyhedral geometry. A polytope is a convex hull, conv $(A)$, of a finite subset $A$ of a finite dimensional real affine space (or vector space) $V$; alternatively, a polytope may be described as a compact intersection of a finite collection of affine half spaces. Here we have chosen Grünbaum's usage [Grü, p. 51] of the term "polytope", instead of the more commonly used "convex polytope", because we shall only be considering polytopes that are convex. If $P$ is a polytope, and $\mathcal{A}$ is a finite collection of affine half spaces such that $P=$ $\cap_{a \in \mathcal{A}} A$, then a face (or cell) of $P$ is a set of the form

$$
P \cap\left(\cap_{A \in \mathcal{A}^{\prime}} \partial A\right),
$$

where $\mathcal{A}^{\prime}$ is a subset of $\mathcal{A}$, and $\partial A$ denotes the bounding hyperplane of $A$ (here we use the convention that $\cap_{A \in \mathcal{A}^{\prime}} \partial A=V$, if $\mathcal{A}^{\prime}=\phi$ ). The dimension of a face is the dimension of its affine span. If $P$ is a polytope, then a facet of $P$ is a face of codimension 1 in $P$. A vertex of $P$ is a face of dimension 0 . Two faces of $P$ are said to be incident if one face is contained in the other. If $S$ is a subset of a polytope $P$, then the carrier, $\operatorname{carr}(S)$, of $S$ is the smallest face of $P$ that contains $S$. If $\sigma$ is a face of $P$, then we shall denote the boundary of $\sigma$ by $\dot{\sigma}$, and we shall denote the relative interior of $\sigma$ by $\sigma-\dot{\sigma}$. If $v \in P$ and $\sigma$ is a face of $P$, then $\sigma=\operatorname{carr}(v)$ if and only if $v$ lies in $\sigma-\dot{\sigma}$. If $S$ is the set of vertices of $P$ that belong to $\sigma$, then the barycenter, $b_{\sigma}=\left(\sum_{s \in S} s\right) /|S|$, of $\sigma$ belongs to $\sigma-\dot{\sigma}$.

If $A$ is a finite subset of a vector space $V$, then, at the risk of sounding pedantic, we shall define a convex combination of elements in $A$ to be a measure $\lambda$ on $V$ such that the total mass of $\lambda$ is 1 , and the support, $\operatorname{supp}(\lambda)$, of $\lambda$ is contained in $A$.

The collection $\mathcal{C}(P)$ of faces of $P$ is a polyhedral complex. That is, $\mathcal{C}(P)$ is a CW complex in the topologists' sense (see, for example, $[\mathbf{L W}]$ or $[\mathbf{W h}]$ ), with cells that are polytopes. Furthermore, the complex $\mathcal{C}(P)$ is regular in the sense that each $\sigma \in \mathcal{C}(P)$ is homeomorphic to a closed ball in a Euclidean space of appropriate dimension. For later use we shall record some properties of the complex $\mathcal{C}(P)$ in the following lemma.

Lemma 2.1. Let $P$ an $n$-dimensional polytope, let $\mathcal{C}(P)$ denote the set of all faces of $P$, and for $-1 \leq k \leq n$, let $\mathcal{C}_{k}$ denote the set of $k$-dimensional faces of $P$. Then $\mathcal{C}(P)$ has the following properties.

(a) If $\sigma, \sigma^{\prime} \in \mathcal{C}(P)$ are incident faces of the same dimension, then $\sigma=\sigma^{\prime}$.

(b) Suppose $\sigma \in \mathcal{C}(P)$, and suppose $A$ is a finite subset of $P$. If $\lambda=$ $\sum_{a \in A} \lambda_{a} a$ is a convex combination of elements in $A$, and $\lambda \in \sigma$, then 
any convex combination of the support, $\operatorname{supp}(\lambda)=\left\{a \in A: \lambda_{a}>0\right\}$, of $\lambda$ is contained in $\sigma$.

(c) If $1 \leq k \leq n$, and $\tau \in \mathcal{C}_{k-2}$ and $\tau^{\prime} \in \mathcal{C}_{k}$ are a pair of incident faces, then there are precisely two $(k-1)$-faces $\sigma_{1}, \sigma_{2} \in \mathcal{C}(P)$ such that $\tau \subset \sigma_{i} \subset \tau^{\prime}$. Note that by (a), $\tau=\sigma_{1} \cap \sigma_{2}$.

The proofs of Parts (a) and (b) routine and will be left to the reader. For a sketch of the proof of Part (c) see 3.1.6 of [Grü], and for a proof that is valid for regular CW complexes see [LW, Theorem III.2.2].

We now touch on some ideas related to the notion of a simplicial complex. A simplicial complex is a pair, $(\mathcal{V}, \mathcal{S})$, where $\mathcal{V}$ is a set, and $\mathcal{S}$ is a collection of finite subsets of $\mathcal{V}$ such that, (a) if $v \in \mathcal{V}$, then $\{v\} \in \mathcal{S}$, and (b) $\tau \in \mathcal{S}$ whenever $\sigma \in \mathcal{S}$ and $\tau \subset \sigma$. The elements of $\mathcal{V}$ are called vertices, and the elements of $\mathcal{S}$ are called simplices. The dimension, $\operatorname{dim} \sigma$, of a simplex $\sigma$ is defined to be $k-1$, where $k$ is the cardinality of $\sigma$. The dimension of $(\mathcal{V}, \mathcal{S})$ is defined to be $\max \{\operatorname{dim} \sigma: \sigma \in \mathcal{S}\}$ or $+\infty$, according as the set $\{\operatorname{dim} \sigma: \sigma \in \mathcal{S}\}$ is, or is not, bounded. If $\tau \subset \sigma$, then the codimension of $\tau$ in $\sigma$ is the cardinality of $\sigma \backslash \tau$. If $\sigma$ is a simplex and $p$ is a point, then the cone with base $\sigma$ and apex $p$ is a simplex, $\sigma * p$, with vertex set equal to a disjoint union of the vertex set of $\sigma$ and $\{p\}$.

Next we shall introduce some terminology that is used in the theory of Coxeter complexes (see [Ti] or [Brow]). A pair of maximal simplices $\sigma, \sigma^{\prime}$ in a simplicial complex are adjacent if the codimensions of $\sigma \cap \sigma^{\prime}$ in $\sigma$ and $\sigma^{\prime}$ are equal to 1 . A chamber complex is a simplicial complex with the property that any two maximal simplices $\sigma, \sigma^{\prime}$ can be joined by a sequence $\sigma=\sigma_{1}, \sigma_{2}, \ldots, \sigma_{k-1}, \sigma_{k}=\sigma^{\prime}$ of maximal simplices, where consecutive simplices are adjacent. The maximal simplices of a chamber complex are called chambers. A chamber complex is thin if every simplex of codimension 1 is contained in precisely two chambers.

We shall show how to construct a simplicial complex from the face complex $\mathcal{C}(P)$ of a polytope $P$. Let $\dot{\mathcal{C}}(P)$ denote the set of proper faces of $P$ :

$$
\dot{\mathcal{C}}(P)=C(p) \backslash\{\phi, P\}
$$

A flag in $P$ is a strictly increasing chain in the poset $(\dot{\mathcal{C}}(P), \subseteq)$. A flag is complete if it is a maximal chain. If $P$ is a polytope, then the set $\mathcal{F}(P)$ of all flags in $P$ is a simplicial complex, the flag complex of $P$; the set of vertices of the complex $\mathcal{F}(P)$ is the set of cells in $P$, and the set of simplices in $\mathcal{F}(P)$ is the set of flags in $P$. The maximal simplices of this simplicial complex are precisely the complete flags in $P$. We now state a key property of $\mathcal{F}(P)$ that 
will be used in the proof of Lemma 4.6, our main lemma in Section 4:

$$
\begin{aligned}
& \text { If } P \text { is a polytope, then the flag complex } \\
& \text { of } \mathcal{F}(P) \text { of } P \text { is a thin chamber complex. }
\end{aligned}
$$

That any two maximal simplices can be connected by a sequence of adjacent maximal simplices follows from an easy argument that involves induction on the dimension of $P$. The assertion that the complex is thin follows from Part (c) of Lemma 2.1 by extending the pair of flags $\tau \subseteq \sigma_{i} \subseteq \tau^{\prime}$ to a pair of complete flags.

Simplicial complexes as described above are sometimes called abstract simplicial complexes. A geometrical simplex is the convex hull of an affinely independent subset of a vector space or affine space. Suppose $\sigma$ is a simplex in a simplicial complex, and suppose the vertices of $\sigma$ constitute an affinely independent subset of some vector space $V$. Then the map: $\tau \mapsto \operatorname{conv}(\tau)$ yields an incidence preserving bijective correspondence between the simplices contained in $\sigma$ and the cells of the geometrical simplex conv $(\sigma)$. The geometrical simplex conv $(\sigma)$ is called a geometric realization or representation of $\sigma$. More generally, if $K=(\mathcal{V}, \mathcal{S})$ is a simplicial complex with a finite vertex set, then there is a geometric realization of $K$ in $\mathbf{R}^{2 n+1}$, where $n=\operatorname{dim} K$. We refer the reader to Section 3.2 of $[\mathbf{S p}]$ for the definition of a geometric realization of a simplicial complex and a proof of the preceding assertion.

We now give an alternate approach to the flag complex $\mathcal{F}(P)$ of a polytope $P$ that will help motivate the proof of Lemma 4.6. If $\sigma \in \mathcal{C}(P)$, then let $\operatorname{sd} \mathcal{C}(P)$ be the simplicial complex $(\mathcal{V}, \mathcal{S})$, where the vertex set $\mathcal{V}$ is the set of barycenters of the simplices in $\mathcal{C}(P)$, and a finite subset $\theta$ of $\mathcal{V}$ belongs to $\mathcal{S}$ if the simplices corresponding to the barycenters in $\theta$ constitute a flag of $P$. The resulting simplicial complex is called the barycentric subdivision or first derived of $\mathcal{C}(P)$. The connection between $\mathcal{F}(P)$ and $\operatorname{sd} \mathcal{C}(P)$ is that

$$
\operatorname{sd} \mathcal{C}(P) \text { is a geometric realization of } \mathcal{F}(P) .
$$

Since we shall only be using (2.3) to motivate the proof of Lemma 4.6, following Brown's example [Brow, p. 28] we shall leave it to the reader to verify its validity.

\section{Coxeter groups.}

We shall now proceed to give an account of some results that we shall need from the theory of Coxeter groups. The reader is referred to the monographs by Bourbaki [Bou], Humphreys [Hu2] or Hiller [Hi] for further information. 
Following Bourbaki [Bou], we define a Coxeter group to be a group $W$ that is equipped with (a) a finite set, $S$, of generators, (b) a symmetric matrix $\left(m_{s s^{\prime}}\right)$ indexed by $S \times S$, with entries that are positive integers or $\infty$, and (c) a presentation of the form

$$
\left\langle s \in S:\left(s s^{\prime}\right)^{m_{s s^{\prime}}}=1, \quad \text { for all } s, s^{\prime} \in S \text { such that } m_{s s^{\prime}} \neq \infty\right\rangle,
$$

where,

$$
m_{s s^{\prime}}=1 \quad \text { if and only if } s=s^{\prime} .
$$

The pair $(W, S)$ is called a Coxeter system, and the matrix $\left(m_{s s^{\prime}}\right)$ is called the Coxeter matrix of $(W, S)$. Suppose $(W, S)$ is a Coxeter system and suppose

$$
V_{S}=\oplus_{s \in S} \mathbf{R} \alpha_{s}
$$

is real vector space with a basis $\left\{\alpha_{s}: s \in S\right\}$ indexed by $S$. Then the canonical bilinear form of $(W, S)$ is the bilinear form of $B$ on $V_{S}$ defined by

$$
B\left(\alpha_{s}, \alpha_{s^{\prime}}\right)=-\cos \left(\pi / m_{s s^{\prime}}\right) .
$$

If $s \in S$, and $\sigma_{s} \in G L\left(V_{s}\right)$ is the mapping defined by

$$
\sigma_{s}(v)=v-2 B\left(v, \alpha_{s}\right) \alpha_{s},
$$

then it can be verified that $\left(\sigma_{s} \sigma_{s^{\prime}}\right)^{m_{s s^{\prime}}}=1$, whenever $s \neq s^{\prime}$. Therefore, there is a representation $\rho: W \rightarrow G L\left(V_{S}\right)$ that satisfies the condition

$$
\rho(s)(v)=\sigma_{s}(v) .
$$

Indeed it turns out that there is a unique representation $\rho_{\text {can }}: W \rightarrow G L\left(V_{S}\right)$ that satisfies (3.1) (see, for example, V.4.3 of [Bou]). This representation is called the canonical (or geometric) representation of $(W, S)$. It is easy to check that the group $\rho_{\text {can }}(W)$ preserves the canonical bilinear form $B$, and so $\rho_{\text {can }}(W)$ is a subgroup of the orthogonal group $O(B) \subset G L\left(V_{S}\right)$ of linear symmetries of $B$.

If $V$ is a finite dimensional real vector space, then an involution $s$ in $G L(V)$ is a linear reflection if the set of fixed points of $s$ has codimension 1 in $V$; equivalently, a linear reflection is an involution in $G L(V)$ that has -1 as an eigenvalue of multiplicity 1. A reflection group is a subgroup of $G L(V)$ that is generated by linear reflections.

We now recall some results from [Cox1] that will be used in the proof of our main result in Section 4. Suppose $\rho: G \subset G L(V)$ is a finite reflection group such that $\{v \in V: g \cdot v=v$, for all $g \in G\}=\{0\}$. Let $\mathcal{R}$ be the set of reflections in $G$. For each $r \in \mathcal{R}$, let $H_{r} \subset V$ be the hyperplane of fixed 
points of $r$, and set $\mathcal{H}=\left\{H_{r}: r \in \mathcal{R}\right\}$. Choose a connected component, $C$, of the hyperplane complement $V \backslash \cup_{H \in \mathcal{H}} H$, and let $D$ be the closure of $C$ in $V$ (the closure is taken here with respect to the standard topology on $V$ ). Then the boundary $D \backslash C$ of $D$ is a union of sets of the form $D \cap H$ (where $H \in \mathcal{H}$ ) that have codimension 1 in $V$. If $H$ is such a hyperplane, then $H$ is said to be a wall of $C$ (in [Cox1] Coxeter used the term "prime" in place of term "wall"). In Lemma 4.5 of [Cox1] Coxeter showed that

$$
D \text { is a fundamental domain for the action of } G \text { on } V \text {. }
$$

In Theorem 8 of [Co1] (see also [Cox1, Lemma 4.6]) he proved that if $S=$ $\left\{s \in \mathcal{R}: H_{s}\right.$ is a wall of $\left.D\right\}$, then

$$
(G, S) \text { is a Coxeter system. }
$$

Using this argument, Coxeter demonstrated that every finite reflection group has a Coxeter presentation. Shortly after that, in [Cox2], he showed that every finite Coxeter group can be realized as a reflection group. Together, these results of Coxeter assert that

$$
\begin{aligned}
& \text { a finite group can be realized as a reflection } \\
& \text { group if and only if it is a Coxeter group. }
\end{aligned}
$$

We shall state two more foundational results in the theory of Coxeter groups. The first is a theorem of Tits [Ti1] (see V.4.4 of [Bou]) that asserts that

$$
\text { the canonical representation } \rho_{\text {can }} \text { of a Coxeter system is faithful, }
$$

and the second result is Witt's Theorem [Wi] (see V.4.8 of [Bou]), which asserts that

$$
W \text { is a finite if and only if } B \text { is positive definite. }
$$

By (3.5) and (3.6), if $W$ is a finite Coxeter group, then the faithful representation $\rho_{\text {can }}: W \hookrightarrow O(B)$ yields an embedding of $W$ in the orthogonal group $O(n)$ of linear symmetries of Euclidean $n$-space, where $n$ is the cardinality of $S$.

In [Cox1], Coxeter used certain graphs that are now called Coxeter graphs to classify all finite reflection groups. Coxeter graphs and closely related objects called Dynkin diagrams arise in classification problems in numerous areas of mathematics. The earliest and possibly the best known of these is the classification of the regular solids, which is attributed to Theaetetus (417-369 B.C.), a contemporary of Plato; needless to say, the classification, as formulated by Theaetetus, did not involve Coxeter groups or 
Dynkin diagrams. A statement of the original result can be found after the final proposition (Proposition 18) in Book XIII of Euclid's Elements [Hea]. (Note that, taken literally, the assertion in Euclid is incorrect because the solids are not assumed to be vertex regular. See [Di, p. 267, Footnote 260] and $[\mathbf{F r W}]$.) The classification of the regular solids is closely related to the problem of classifying finite subgroups of $S U(2)$ and $S O(3)$. According to Coxeter ([Cox3, pp. 56-57]) the finite subgroups of $S O(3)$ were first classified by Hessel in 1830, but remained unnoticed until it was published in 1897 [Hes]. Other treatments of the classification of platonic solids and the finite subgroups of $S U(2)$ and $S O(3)$ can be found in [Schw], [Kl], [Cox3], $[\mathbf{L a}],[\mathbf{L y}]$, and $[\mathbf{R}]$.

Additional classifications in which Coxeter graphs and Dynkin diagrams arise include Lie theory $[\mathbf{K i}],[\mathbf{C a}],[\mathbf{B o u}],[\mathbf{H u 1}],[\mathbf{F u H}],[\mathbf{K n}]$, regular polytopes [Schl], [Cox3], [Berg], singularity theory [Du V], [Ar1], [Ar2], [ArGV1], [ArGV2], [La], invariant theory [K1], [Ch], [Se1], representations of algebras [Ga1], [Ga2], [BernGP], sphere packing [KorZ], [Bl], [Mi], [ConS], graphs with norm less than 2 [Sm], [BrouCN], [GoHJ], von Neumann algebras [GoHJ], conformal field theories [CaIZ], and gravitational instantons [Kr1], [Kr2], [At2].

Not all of the Dynkin diagrams corresponding to finite Coxeter groups occur in each of the classification problems listed above. Those that occur most frequently are the diagrams of types $A, D$, and $E$. These correspond to Coxeter groups with canonical embeddings $\rho_{\text {can }}: W \hookrightarrow G L\left(V_{S}\right)$ that stabilize the $\mathbf{Z}$-span of a basis of $V_{S}$ that consists of vectors of equal length. See the survey articles [HazHSV], [Dur], [Sl], [Gi], the monograph [La], and Slodowy's commentary in the most recent edition [Kl] of Klein's classic work on the icosahedron for additional information on various classification problems that have solutions involving Dynkin diagrams and some connections between these seemingly unrelated areas of mathematics.

\section{Eaton's Criterion, the Convolution Theorem, and Coxeter groups.}

In this section we shall prove that if a finite group $G$ satisfies the Convolution Theorem, then $G$ is a Coxeter group.

If $G$ is a finite group, then a finite dimensional real representation $\rho$ : $G \rightarrow G L(V)$ may be viewed as a finitely generated module of the real group algebra $\mathbf{R}[G]$. Conversely every finitely generated $\mathbf{R}[G]$-module can be viewed as a finite dimensional real representation of $G$ (see, for example, [Se2, Section 6.1]). We shall frequently use this correspondence between representations of a group and modules of the corresponding group algebra. 
If $A$ is a subset of a finite group $G$, then $\operatorname{conv}(A)$ will always denote the convex hull of $A$ in the group algebra $\mathbf{R}[G]$ of $G$, and, if $k \in \operatorname{conv}(G)$, then $\operatorname{supp}(k)$ will always denote the support of $k$ viewed as a convex combination of elements in $G$.

Let $\rho: G \hookrightarrow G L(V)$ be a finite group, and let $P \subset V$ be a polytope of the form $\operatorname{conv}_{\rho}(v)$, for some $v \in V$. We observe that the action of $G$ on $V$ induces an action $\phi: G \times P \rightarrow P$ of $G$ on $P$ via automorphisms of $\mathcal{C}(P)$ (i.e., bijections from $\mathcal{C}(P)$ to itself that preserve incidence and dimension). This action $\phi$ extends to an action $\psi: \operatorname{conv}(G) \times P \rightarrow P$ of $\operatorname{conv}(G)$ on $P$. Suppose $u \in \sigma-\dot{\sigma}$, for some $\sigma \in \mathcal{C}(P)$. If $k \in \operatorname{conv}(G)$ and $\tau=\operatorname{carr}(k \cdot u)$, then by Part (b) of Lemma 2.1, $g \cdot u \in \tau$ for every $g \in \operatorname{supp}(k)$. Since $G$ acts on $P$ via automorphisms of $\mathcal{C}(P)$, it follows that $\operatorname{carr}(g \cdot u)=g \cdot \operatorname{carr}(u)=g \cdot \sigma$, for every $g \in \operatorname{supp}(k)$. Therefore $g \cdot \sigma \subset \tau$, for every $g \in \operatorname{supp}(k)$. Since $\operatorname{dim} g \cdot \sigma=\operatorname{dim} \sigma$, we see that

$$
\operatorname{dim} \sigma \leq \operatorname{dim} \tau
$$

In the following lemma we shall focus on the case where $\operatorname{dim} \sigma=\operatorname{dim} \tau$. If $\sigma \in \mathcal{C}(P)$, then we shall denote the stabilizer of $\sigma$ in $G$ by $G_{\sigma}$.

Lemma 4.2. Suppose $\rho: G \hookrightarrow G L(V)$ is a finite linear group. Let $P \subset V$ be a polytope of the form $\operatorname{conv}_{\rho}(v)$, for some $v \in V$, let $k \in \operatorname{conv}(G) \subset \mathbf{R}[G]$, let $\sigma \in \mathcal{C}(P)$, and let $\tau$ be the carrier of $k \cdot u$, for some $u \in \sigma-\dot{\sigma}$. Then the following assertion are equivalent.

(a) $\operatorname{dim} \sigma=\operatorname{dim} \tau$.

(b) $\tau=g \cdot \sigma$, for every $g \in \operatorname{supp}(k)$.

(c) $\operatorname{supp}(k) \subset g G_{\sigma}$, for every $g \in \operatorname{supp}(k)$.

Furthermore, if $k, k^{\prime} \in \operatorname{conv}(G)$ and $\operatorname{carr}\left(k^{\prime} k \cdot u\right)=\sigma$, then $\operatorname{supp}\left(k^{\prime}\right) \subset G_{\sigma} g^{-1}$ and $\operatorname{supp}(k) \subset g G_{\sigma}$, for every $g \in \operatorname{supp}(k)$.

Proof. As noted above, since $G$ acts on $P$ via automorphisms of $\mathcal{C}(P)$,

$$
g \cdot \sigma=\operatorname{carr}(g \cdot u) \subset \operatorname{carr}(k \cdot u)=\tau, \quad \text { for every } g \in \operatorname{supp}(k) .
$$

Now assume that (a) is true. Then $\operatorname{dim}(g \cdot \sigma)=\operatorname{dim} \sigma=\operatorname{dim} \tau$, and therefore by Part (a) of Lemma 2.1 we see that in fact

$$
g \cdot \sigma=\tau, \quad \text { for every } g \in \operatorname{supp}(k) \text {. }
$$

We have thus shown that (a) implies (b). That (b) implies (a) is an immediate consequence of the observation that the action of $g$ preserves dimensions in $\mathcal{C}(P)$, for every $g \in \operatorname{supp}(k)$. 
Assertion (c) is merely a slight reformulation of (b) that will be of use to us later. The proof of the equivalence of (b) and (c) will be left to the reader. The final assertion of the lemma follows from the equivalence of (a) and (c). Again, the proof will be left to the reader.

In Proposition 4.10 and 4.11 of his paper [E2], Eaton established a necessary condition for a compact group to satisfy the Convolution Theorem. If $\rho: G \hookrightarrow G L(V)$ is a linear group, then we shall say that $\rho$ satisfies Eaton's Criterion if

$$
\operatorname{conv}_{\rho}\left[x+\operatorname{conv}_{\rho} y\right] \text { is a convex subset of } V, \text { for all } x, y \in V \text {; }
$$

that is if, for all $x, y \in V$, all $k_{1}^{\prime}, k_{2}^{\prime}, k_{1}^{\prime \prime}, k_{2}^{\prime \prime} \in \operatorname{conv}(G)$, and every $t \in(0,1)$, there exist $k_{1}, k_{2} \in \operatorname{conv}(G)$ such that

$$
t k_{1}^{\prime} \cdot\left[x+k_{2}^{\prime} \cdot y\right]+(1-t) k_{1}^{\prime \prime} \cdot\left[x+k_{2}^{\prime \prime} \cdot y\right]=k_{1} \cdot\left[x+k_{2} \cdot y\right] .
$$

Let $\rho: G \rightarrow G L(V)$ be a representation of a group, and let $V^{G}$ denote the set

$$
\{v \in V: g \cdot v=v, \quad \text { for all } g \in G\}
$$

of $G$-invariants in $V$. Then $V^{G}$ is an $\mathbf{R}[G]$-submodule of the $\mathbf{R}[G]$-module $V$. We shall say that the action of $G$ on $V$ is essential if $V^{G}=0$. Now if $G$ is finite, then by Maschke's Theorem ([Se2, Proposition 6.1.9]), there is an $\mathbf{R}[G]$-module $\widetilde{V}$ that is a complement to $V^{G}$ in $V$. The module $\widetilde{V}$ is the sum of the irreducible submodules of $V$ on which $G$ acts essentially, and so it is in fact the only $\mathbf{R}[G]$-submodule of $V$ that is a complement of $V^{G}$ in $V$. We shall call $\widetilde{V}$ the essential part of $V$, and we shall call the subrepresentation $\tilde{\rho}: G \rightarrow G L(\widetilde{V})$ of $\rho$ the essential part of $\rho$. One checks easily that $V$ satisfies Eaton's Criterion if and only if $\widetilde{V}$ satisfies Eaton's Criterion. For this reason we shall frequently confine our attention to $\mathbf{R}[G]$-modules $V$ on which the action of $G$ is essential.

If $u \in V$, then we shall say that $u$ is $\rho$-regular, if the stabilizer $G_{u}=\{g \in$ $G: g \cdot u=u\}$ of $u$ in $G$ is the identity subgroup of $G$.

Lemma 4.4. Suppose $\rho: G \hookrightarrow G L(V)$ is a finite essential linear group that satisfies Eaton's Criterion. Then the $\mathbf{R}[G]$-module $V$ is cyclic and is generated by any $\rho$-regular element in $V$.

Proof. We begin by noting that if $g$ is a nontrivial element of $G$, then the subspace, $V^{g}$, of points in $V$ that are fixed by $g$ has positive codimension and hence empty interior in $V$, because $\rho$ is a faithful representation. Therefore, by the finiteness of $G$, the set $\cup_{g \in G \backslash\{1\}} V^{g}$ of non-regular elements of $V$ is a proper subset of $V$, and so $V$ contains a $\rho$-regular element $u$. Let $U$ be 
the cyclic $\mathbf{R}[G]$-submodule of $V$ that is generated by $u$. We shall prove that $V=U$.

Suppose $U$ is a proper submodule of $V$. By the complete reducibility of $V$, the $G$-module $U$ has a complement, $W$, in $V$. Choose some nonzero $w \in W$. Since $\rho$ is essential and $w \in V$ is nonzero, there is a $g \in G$ such that $g \cdot w \neq w$. Now $u+w$ and $u+g \cdot w=g \cdot\left(w+g^{-1} \cdot u\right)$ belong to $\operatorname{conv}_{\rho}\left[w+\operatorname{conv}_{\rho} u\right]$. Therefore, given $t \in(0,1)$, there exist $k^{\prime}=k^{\prime}(t)$ and $k=k(t)$ in $\operatorname{conv}(G)$ such that

$$
u+t w+(1-t) g \cdot w=k^{\prime} \cdot[w+k \cdot u] .
$$

The independence of $U$ and $W$ implies that

$$
t w+(1-t) g \cdot w=k^{\prime} \cdot w
$$

and

$$
k^{\prime} k \cdot u=u \text {. }
$$

Since the stabilizer $G_{u}$ of $u$ is trivial it follows that $k^{\prime} k=1$, and consequently $k^{\prime} \in G$ by the final assertion of Lemma 4.2. But $g \cdot w \neq w$ by assumption, and therefore (4.5) implies that $t \mapsto k^{\prime}(t)$ is an injective map from $(0,1)$ to $G$, thus contradicting the assumption that $G$ is finite. Hence $U$ is not a proper submodule of $V$. This means that $V=U$, and so we conclude, as desired, that $V$ is a cyclic module.

In preparation for our main result, Theorem 4.11, we shall now establish the existence of certain reflections in a finite essential linear group that satisfies Eaton's Criterion. Much of the work that goes into establishing Theorem 4.11 will in fact be carried out in the following proof.

Lemma 4.6. Let $\rho: G \hookrightarrow G L(V)$ be a finite essential linear group that satisfies Eaton's Criterion. Let $v$ be a $\rho$-regular element in $V$, let $P$ denote the polytope $\operatorname{conv}_{\rho}(v)$, and let $v^{\prime}$ be a vertex of $P$ that is adjacent to $v$. Then there is an involution $s$ in $G$ such that $\rho(s)$ is a linear reflection that maps $v$ to $v^{\prime}$.

Proof. We begin by introducing some notation. Let $I$ denote the set $\{0, \ldots$, $n-1$, let $\mathcal{F}(P)$ be the flag complex of the polytope $P$, and let $C$ and $C^{\prime}$ be a pair of adjacent chambers in $\mathcal{F}(P)$ such that $C \backslash C^{\prime}=v$ and $C^{\prime} \backslash C=v^{\prime}$. Set $C \cap C^{\prime}=\{\sigma(1), \ldots, \sigma(n-1)\}$, where, for each $i \in I$, the dimension of $\sigma(i)$ is $i$.

Recall that in Lemma 4.2 we studied the action of $\operatorname{conv}(G)$ on $\mathcal{C}(P)$. This action induces an action of $\operatorname{conv}(G)$ on $\mathcal{F}(P)$. We shall use Eaton's Criterion and the action on $\mathcal{F}(P)$ to deduce the existence of a reflection that takes $v$ to $v^{\prime}$. Since we shall be using Eaton's Criterion in the form (4.3), we shall find 
it convenient to have $G$ act on the barycentric subdivision $\operatorname{sd} \mathcal{C}(P)$, which is a geometric realization of $\mathcal{F}(P)$ by $(2.3)$, rather than on $\mathcal{F}(P)$ itself.

Let $b_{i}$ be the barycenter of $\sigma(i)$, and, for the sake of uniformity, set $b_{0}=v$ and $b_{0}^{\prime}=v^{\prime}$. For each positive $i \in I$, let $\beta(i)$ denote the simplex $\left\{b_{j}: 0<\right.$ $j \leq i\}$, and let $b_{\beta(i)}$ denote the barycenter of $\beta(i)$. Under the correspondence between the simplices of $\operatorname{sd} \mathcal{C}(P)$ and those of $\mathcal{F}(P), \beta(i)$ corresponds to the flag $\sigma(1) \subset \cdots \subset \sigma(i)$. If $t \in[0,1]$, then let $p(t)$ be the point $t b_{0}+(1-t) b_{0}^{\prime}$, let $\beta(i) * p(t)$ be the cone over $\beta(i)$ with apex $p(t)$, and let $b_{\beta(i) * p(t)}$ denote the barycenter of $\beta(i) * p(t)$. We note that

$$
b_{\beta(i) * p(t)}=[1 /(i+1)]\left[t b_{0}+(1-t) s \cdot b_{0}\right]+[i /(i+1)] b_{\beta(i)} .
$$

Setting $t=0,1$ and $i=n-1$ we see by the definition of $\operatorname{conv}_{\rho}[\cdot]$ that

$$
b_{\beta(n-1) * p(0)}, b_{\beta(n-1) * p(1)} \in \operatorname{conv}_{\rho}\left[(1 / n) b_{0}+\operatorname{conv}_{\rho}\left[(1-(1 / n)) b_{\beta(n-1)}\right]\right] .
$$

We shall now consider a fixed $t \in(0,1)$ and simplify notation by denoting $p(t)$ by $p$. Since $\rho$ satisfies Eaton's Criterion and $b_{\beta(n-1) * p}$ is a convex combination of $b_{\beta(n-1) * p(0)}$ and $b_{\beta(n-1) * p(1)}$, by (4.7) there are elements $k_{1}, k_{2}$ in $\operatorname{conv}(G)$ such that

$$
b_{\beta(n-1) * p}=(1 / n) k_{1} \cdot b_{0}+[(n-1) / n] k_{1} k_{2} \cdot b_{\beta(n-1)} .
$$

Observe that the left hand side of the preceding equation belongs to the facet $\sigma(n-1)$ of $P$. Therefore, noting that $b_{\beta(n-1)}=\left(\sum_{j=1}^{n-1} b_{j}\right) /(n-1)$, we deduce by Part (b) of Lemma 2.1 that

$$
k_{1} k_{2} \cdot b_{n-1} \in \sigma(n-1) .
$$

By Lemma 4.2 it follows that $\operatorname{supp}\left(k_{1} k_{2}\right) \subset G_{\sigma(n-1)}$. But $g \cdot b_{n-1}=b_{n-1}$, for every $g \in G_{\sigma(n-1)}$, because $b_{n-1}$ is the barycenter of $\sigma(n-1)$, and so

$$
k_{1} k_{2} \cdot b_{n-1}=b_{n-1} .
$$

In light of this, by projecting $\beta(n-1) * p$ onto $\beta(n-2) * p$ along rays emanating from $b_{n-1}$, we deduce from (4.8) that

$$
b_{\beta(n-2) * p}=[1 /(n-1)] k_{1} \cdot b_{0}+[(n-2) /(n-1)] k_{1} k_{2} \cdot b_{\beta(n-2)} .
$$

Repeating the argument above we get

$$
k_{1} k_{2} \cdot b_{i}=b_{i}, \quad \text { for all } i>0,
$$

and

$$
b_{\beta(i) * p}=[1 /(i+1)] k_{1} \cdot b_{0}+[i /(i+1)] k_{1} k_{2} \cdot b_{\beta(i)}, \quad \text { for all } i>0 .
$$


In particular, when $i=1$, we have

$$
b_{\beta(1) * p}=\frac{1}{2} k_{1} \cdot b_{0}+\frac{1}{2} k_{1} k_{2} \cdot b_{\beta(1)} .
$$

Now $k_{1} k_{2} \cdot b_{\beta(1)}=b_{\beta(1)}$ by $(4.9)$, and so

$$
b_{\beta(1) * p}=\frac{1}{2} k_{1} \cdot b_{0}+\frac{1}{2} b_{\beta(1)} .
$$

Since

$$
b_{\beta(1) * p}=\frac{1}{2} p+\frac{1}{2} b_{\beta(1)}
$$

by definition, we deduce that

$$
k_{1} \cdot b_{0}=p=t b_{0}+(1-t) b_{o}^{\prime} .
$$

This means that $k_{1} \cdot b_{0}$ belongs to the edge of $P$ that joins the adjacent vertices $b_{0}$ and $b_{0}^{\prime}$. This in turn implies that $g \cdot b_{0}=b_{0}$ or $b_{0}^{\prime}$, for every $g \in \operatorname{supp}\left(k_{1}\right)$. In addition, since $t$ lies strictly between 0 and 1 , there exist elements $g_{1}, g_{2}$ in $\operatorname{supp}\left(k_{1}\right)$ such that $g_{1} \cdot b_{0}=b_{0}$ and $g_{2} \cdot b_{0}=b_{0}^{\prime}$. Observe that, by (4.9) and the final assertion of Lemma 4.2, $g_{2} g_{1}^{-1} \in G_{\sigma(i)}$ for all $i>0$. Hence, if $s=g_{2} g_{1}^{-1}$, then

$$
s \cdot \sigma(i)=\sigma(i), \quad \text { for all } i>0 \text {. }
$$

In other words,

$$
s \cdot\left(C \cap C^{\prime}\right)=C \cap C^{\prime} .
$$

Since $C \cap C^{\prime}=s \cdot\left(C \cap C^{\prime}\right)$ is a simplex of codimension 1 in the flag complex $\mathcal{F}(P)$ of $P$, and $\mathcal{F}(P)$ is a thin chamber complex by $(2.2)$, it follows that $C$ and $C^{\prime}$ are the only chambers in $\mathcal{F}(P)$ that contain $C \cap C^{\prime}$. Likewise, $s \cdot C$ and $s \cdot C^{\prime}$ are the only chambers in $\mathcal{F}(P)$ that contain $s \cdot\left(C \cap C^{\prime}\right)=C \cap C^{\prime}$; thus

$$
\left\{s \cdot C, s \cdot C^{\prime}\right\}=\left\{C, C^{\prime}\right\} .
$$

Now $s \cdot C \neq C$, because $s \cdot b_{0}=b_{0}^{\prime}$, and so $s \cdot C=C^{\prime}$ and $s \cdot C^{\prime}=C$. Next, observe that $s^{2}$ fixes the subset $\left\{b_{i}: i \in I\right\}$ of $V$ pointwise, as $s^{2} \cdot C=s \cdot C^{\prime}=$ $C$. By Lemma 4.4, the module $V$ is cyclic with generator $v$, and therefore the set $\left\{b_{i}: i \in I\right\}$ spans $V$. Hence $s^{2}=1$. Moreover, since $s$ fixes $b_{i}$ for each $i>0$, and $s \cdot v=g_{2} g_{1}^{-1} \cdot b_{0}=b_{0}^{\prime}=v^{\prime}$, the kernel of $s-\mathrm{id}_{V}$ has codimension 1 in $V$. This means that $s$ is a linear reflection such that $v^{\prime}=s \cdot v$, thus concluding the proof. 
The following result, together with the work of Eaton [E2], and Eaton and Perlman [EP1], will yield the main result, Theorem 4.11, of this paper.

Proposition 4.10. Suppose $\rho: G \hookrightarrow G L(V)$ is a finite linear group that satisfies Eaton's Criterion. Then $G$ is a Coxeter group, and the essential part of $V$ is isomorphic to the canonical Coxeter module of $G$.

Proof. Since $\rho$ satisfies Eaton's Criterion, the essential part $\tilde{\rho}$ of $\rho$ also satisfies Eaton's Criterion. In the proof of the first assertion of the proposition we shall assume therefore that $\rho$ is essential. Let $\mathcal{R}$ denote the collection of all reflections in $G$. By the preceding lemma, $\mathcal{R}$ is a nonempty set. Let $W$ denote the subgroup of $G$ that is generated by $\mathcal{R}$. We shall prove that $G=W$.

To see this, choose some $\rho$-regular $v \in V$, let $P$ be the polytope $\operatorname{conv}_{\rho}(v)$, and let $g$ be an arbitrary element of $G$. Then $g \cdot v$ is a vertex of $P$, and there is a sequence $\left\{\left[v_{i}, v_{i+1}\right]: 0 \leq i \leq k-1\right\}$ of edges of $P$ that connect $v_{0}=v$ and $v_{k}=g \cdot v$. By Lemma 4.6, there is a sequence $\left\{r_{i}: 0 \leq i \leq k-1\right\}$ of reflections in $W$ such that $v_{i+1}=r_{i} \cdot v_{i}$, for $0 \leq i \leq k-1$. Let $w=r_{k-1} \cdots r_{1} r_{0}$. Then $w \in W$, and $g \cdot v=w \cdot v$. Since $w^{-1} g$ fixes the $\rho$-regular element $v$, it follows that $w^{-1} g=1$. We see therefore that an arbitrarily chosen element $g$ of $G$ belongs to $W$. This means that $G=W$ is a finite reflection group, and so, by (3.4), $G$ is a Coxeter group.

It remains to show that $\widetilde{V}$ is isomorphic to the canonical Coxeter module of $G$. Let $S$ be the collection of reflections across the walls of a fundamental domain, $D$, of the sort described in (3.2). Then, by $(3.3),(G, S)$ is a Coxeter system. Furthermore the maps $\tilde{\rho}(s)$, where $s \in S$, act as reflections across the walls of $D$, and $\tilde{\rho}: G \rightarrow G L(\tilde{V})$ satisfies (3.1). Since $\rho_{\text {can }}: W \rightarrow$ $G L\left(V_{S}\right)$ is characterized by $(3.1)$, by identifying $\widetilde{V}$ with $V_{S}$ we see that the representation $\tilde{\rho}$ is equivalent to the canonical representation of $(G, S)$. This completes the proof.

We now state the main result of this paper.

Theorem 4.11. Suppose $\rho: G \hookrightarrow G L(V)$ is a finite linear group. Then the conditions,

(a) $\rho$ is a Coxeter group,

(b) $\rho$ satisfies the Convolution Theorem, and

(c) $\rho$ satisfies Eaton's Criterion

are equivalent. If these conditions are satisfied, then $\rho$ decomposes into a direct sum $\rho_{\text {can }} \oplus \rho_{\text {triv }}$, where $\rho_{\text {can }}$ is the canonical Coxeter representation of $G$, and $\rho_{\text {triv }}: G \rightarrow G L\left(V^{G}\right)$ is the trivial representation of $G$ on the set $V^{G}$ 
of $G$-invariants of $V$.

Proof. By Theorem 5.1 of [EP1], Assertion (a) implies Assertion (b). Next, by Proposition 4.10 and 4.11 of [E2], Assertion (b) implies Assertion (c). That Assertion (c) implies Assertion (a) follows from Proposition 4.10 above.

If Assertions (a), (b) and (c) are satisfied, then by Proposition 4.10 the essential part of $\rho$ is the canonical Coxeter representation $\rho_{\text {can }}$ of $G$, and the complement of $\rho_{\text {can }}$ in $\rho$ is necessarily the trivial representation of $G$ on the set $V^{G}$ of $G$-invariants of $V$. This concludes the proof.

\section{References}

[Ad] J.F. Adams, Lectures on the Lie groups, W.A. Benjamin, New York, 1969.

[An] T.W. Anderson, The integral of symmetric unimodal function over a symmetric convex set and some probability inequalities, Proc. Amer. Math. Soc., 6 (1955), 170-176.

[Ar1] V.I. Arnol'd, Normal forms of functions near degenerate critical points, Weyl groups $A_{k}, D_{k}, E_{k}$ and Lagrangian singularities, Funktsional. Anal. i Prilozhen., 6 (1972), 3-25.

[Ar2] - Critical points of functions on a manifold with boundary, the simple Lie groups $B_{k}, C_{k}$ and $F_{k}$ and singularities of evolutes, Uspekhi Mat. Nauk, 33 (1978), 91-105.

[ArGV1] V.I. Arnol'd, S.M. Gusĕ̌n-Zade and A.N. Varchenko, Singularities of differentiable maps, Vol. I, Nauka, Moscow, 1982; English translation, Birkhäuser, Boston, 1988.

[ArGV2] _ Singularities of differentiable maps, Vol. II, Nauka, Moscow, 1982; English translation, Birkhäuser, Boston, 1988.

[At1] M.F. Atiyah, Convexity and commuting Hamiltonians, Bull. Lond. Math. Soc., 14 (1982), 1-15.

[At2] , Hyper-Kähler manifolds, in "Complex geometry and analysis", Lectures Notes in Mathematics, Vol. 1422, Springer-Verlag, Berlin-Heidelberg-New York, (1990), 1-13.

[AtB] M.F. Atiyah and R. Bott, The Yang-Mills equations over Riemann surfaces, Phil. Trans. R. Soc. Lond., 308(A) (1982), 523-615.

[Berg] M. Berger, Geometry II, Springer-Verlag, Berlin-Heidelberg-New York.

[BernGP] I.N. Bernstein, I.M. Gel'fand and V.A. Ponomarev, Coxeter functors and Gabriel's theorem, Uspekhi Mat. Nauk, 28 (1973), 19-33.

[Bl] H.F. Blichfeldt, The minimum values of quadratic forms in six, seven, and eight variables, Math. Zeits., 39 (1935), 1-15.

[Bor1] A. Borel, Some remarks about Lie groups transitive on spheres and tori, Bull. Amer. Math. Soc., 53 (1949), 580-587.

[Bor2] Le plan projectif des octaves et les spheres comme espace homogènes, C. R. Acad. Sc. Paris, 230 (1950), 1378-1380.

[Bou] N. Bourbaki, Groupes et algèbres de Lie, Masson, Paris, 1981. 
[BrouCN] A.E. Brouwer, A.M. Cohen and A. Neumaier, Distance-regular graphs, SpringerVerlag, Berlin-Heidelberg-New York, 1989.

[Brow] K. Brown, Buildings, Springer-Verlag, Berlin-Heidelberg-New York, 1989.

[CaIZ] A. Capelli, C. Itzykson and J.B. Zuber, The A-D-E classification of minimal and $A_{1}^{(1)}$ conformal invariant theories, Communications in Math. Phys., 113 (1987), $1-16$.

[Ca] É. Cartan, Sur la structure des groupes de transformations finis et continus, Thése, Nony, Paris, 1894.

[Ch] C. Chevalley, Invariants of finite reflection groups, Amer. J. Math., 67 (1955), 778-782.

[ConS] J.E. Conway and N.J.A. Sloane, Sphere packings, lattices and groups, SpringerVerlag, Berlin-Heidelberg-New York, 1991.

[Cox1] H.S.M. Coxeter, Discrete groups generated by reflections, Ann. of Math., 35 (1934), 588-621.

[Cox2] The complete enumeration of finite groups of the form $R_{i}^{2}=\left(R_{i} R_{j}\right)^{k_{i j}}=1$, J. Lond. Math. Soc., 10 (1935), 21-25.

[Cox3] , Regular polytopes, Dover, New York, 1973.

[Di] E.J. Dijksterhuis, De elementen van Euclides, Vol. 2, P. Noordhoff, Groningen, 1930.

[Du V] P. Du Val, On isolated singularities which do not affect the conditions of adjunction, Part I, Proc. Cambridge Phil. Soc., 30 (1934), 453-465.

[Dur] A.H. Durfee, Fifteen characterizations of rational double points and simple critical points, L'Enseignement Mathématique , 25 (1979), 131-163.

[E1] M.L. Eaton, Lectures on topics in probability inequalities, Centrum voor Wiskunde en Informatica, Amsterdam, 1987.

[E2] - On group induced orderings, monotone functions, and convolution theorems, in "Inequalities in Statistics and Probability", IMS Lecture Notes-Monograph Series, 5 (1984), 13-25.

[EP1] M.L. Eaton and M.D. Perlman, Reflection groups, generalized Schur functions, and the geometry of majorization, Ann. Prob., 5 (1977), 829-860.

[EP2] - Generating $O(n)$ with reflections, Pac. J. Math., 73 (1977), 73-80.

[FrW] H. Freudenthal and B.L. van der Waerden, Over een bewering van Euclides, Simon Stevin, 25 (1947), 115-121.

[FuH] W. Fulton and J. Harris, Representation Theory: A First Course, Springer-Verlag, Berlin-Heidelberg-New York, 1991.

[Ga1] P. Gabriel, Unzerlegbare darstellungen I, Manuscripta Math., 6 (1972), 71-103.

[Ga2] - Indecomposable representations II, Simposia Math. Inst. Naz. Alta Mat., 11 (1973), 81-104.

[Gi] A.B. Givental', Reflection groups in singularity theory, in "Singularity Theory and Some Problems of Functional Analysis", A.M.S. Translations, Series 153(2) (1992), 39-71.

[GoHJ] F.M. Goodman, P. de la Harpe and V.F.R. Jones, Coxeter graphs and towers of algebras, Mathematical Sciences Research Institute Publications, Vol. 14, SpringerVerlag, Berlin-Heidelberg-New York, 1989. 
[GraY] J.H. Grace and A. Young, The algebra of invariants, reprinted 1978, Chelsea Publishing Company, Bronx, N.Y., 1903.

[Grü] B. Grünbaum, Convex polytopes, John Wiley \& Sons, London, 1967.

[GuS] V. Guillemin and S. Sternberg, Convexity properties of the moment mapping, Invent. Math., 67 (1982), 491-513.

[HarLP] G.H. Hardy, J.E. Littlewood and G. Polya, Inequalities, Cambridge Univ. Press, London and New York, 1952.

[HazHSV] M. Hazewinkel, W. Hesselink, D. Siersma and F.D. Veldkamp, The ubiquity of Coxeter-Dynkin diagrams (an introduction to the A-D-E problem), Nieuw Arch. Wiskunde, 25(III) (1977), 257-307.

[Hea] T.L. Heath, The thirteen books of Euclid's elements, Vol. III, Dover, New York, 1956.

[Hes] J.F.C. Hessel, Krystallometrie oder Krystallonomie und Krystallographie, (Ostwald's Klassiker der exakten Wissenschaften, 88, 89), 1897.

[Hi] H. Hiller, Geometry of Coxeter groups, Research Notes in Mathematics, No. 54, Pitman, Boston, 1982.

[Ho] A. Horn, Double stochastic matrices and the diagonal of a rotation matrix, Amer. J. Math., 76 (1954), 620-630.

[Hu1] J.E. Humphreys, Introduction to Lie algebras and representation theory, SpringerVerlag, Berlin-Heidelberg-New York, 1980.

[Hu2] , Reflection groups and Coxeter groups, Cambridge Univ. Press, Cambridge, U.K., 1990.

[Ke] J.H.B. Kemperman, Review of "Inequalities: Theory of majorization and its applications", Bull. Amer. Math. Soc., 5 (1981), 319-324.

[Ki] W. Killing, Die Zusammensetzung der stetigen endlichen Transformation-gruppen I-IV, Math. Annalen, 31, 33, 34, 36 (1888-1890).

[Kl] F. Klein, Vorlesungen über das Ikosaeder und die Ausflösung der Gleichungen vom fünften Grade, reprint of the 1884 original. Edited, with an introduction and commentary by P. Slodowy, Birkhäser Verlag, Basel, 1993 (English trans. Lectures on the icosahedron and the solution of equations of the fifth degree, Dover, Boston, 1956).

[Kn] A.W. Knapp, Lie groups beyond an introduction, Birkhäser, Boston, 1996.

[KorZ] A. Korkine and G. Zolotareff, Sur les formes quadratique positives, Math. Ann., 11 (1877), 242-292.

[Kos] B. Kostant, On convexity, the Weyl group and the Iwasawa decomposition, Ann. Sci. Ec. Norm. Sup., 6 (1973), 413-455.

[Kr1] P.B. Kronheimer, The construction of ALE spaces as hyper-kähler quotients, J. Differential Geometry, 29 (1989), 665-683.

[Kr2] _ A Torelli-type theorem for gravitational instantons, J. Differential Geometry, 29 (1989), 685-697.

[La] K. Lamotke, Regular solids and isolated singularities, Friedr. Vieweg \& Sohn, Braunschweig, 1986.

[LW] A.T. Lundell and S. Weingram, The topology of $C W$ complexes, Van Nostrand Reinhold, New York, 1969. 
[Ly] R.C. Lyndon, Groups and geometry, Cambridge University Press, Cambridge, U.K., 1980 .

[MaO1] A.W. Marshall and I. Oklin, Inequalities: Theory of majorization and its applications, Academic Press, New York, 1979.

[MaO2] Majorization in multivariate distributions, Ann. Statist., 2 (1974), 11891200.

[Mi] J. Milnor, Hilbert's Problem 18: On crystalographic groups, fundamental domains, and sphere packing, in "Mathematical developments arising from Hilbert problems", Proc. Symp. Pure Math., 28 (1976), 491-506.

[MoS] D. Montgomery and H. Samelson, Transformation groups of spheres, Ann. of Math., 44 (1943), 454-470.

[Mud] G.S. Mudholkar, The integral of an invariant unimodal function over an invariant convex set: An inequality and applications, Proc. Amer. Math. Soc., 17 (1966), 1327-1333.

[Mui] R.F. Muirhead, Some methods applicable to identities and inequalities of symmetric algebraic functions of $n$ letters, Proc. Edinburg Math. Soc., 21 (1903), 144-157.

[P] M.D. Perlman, T.W. Anderson's theorem on the integral of a symmetric unimodal function over a symmetric convex set and its applications in probability and statistics, in "Collected Papers of T.W. Anderson", (G. Styan, ed.), Wiley, (1990), 16271641.

[R] E.G. Rees, Notes on geometry, Springer-Verlag, Berlin-Heidelberg-New York, 1988.

[Schl] L. Schläfli, Theorie der vielfachen Kontinuität, Denkschriften der Schweizerischen Naturforschenden Gesellschaft, 38 (1901), 1-237.

[Schu] I. Schur, Über eine Klasse von Mittelbildungen mit Anwendungen auf Determinantentheorie, Sitzungsberichte der Berliner Mathematischen Gesellschaft, 22 (1923), 9-20.

[Schw] H.A. Schwarz, Ueber diejenigen Fälle, in welchen die Gaussische hypergeometrische Reiheeine algebraische Function ihres vierten Elements darstellt, J. Reine Angew. Math., 75 (1872).

[Se1] J.-P. Serre, Groupes finis d'automorphisms d'anneaux locaux règulieres, in "Colloq. d'Algébre", Secrétariat Mathématique, Paris, 1968.

[Se2] , Linear representation of finite groups, Graduate Texts in Mathmatics, No. 2, Springer-Verlag, Berlin-Heidelberg-New York, 1977.

[Sh] S. Sherman, A theorem on convex sets with applications, Ann. Math. Statist., 6 (1955), 926-1931.

[Sl] P. Slodowy, Platonic solids, Kleinian singularities, and Lie groups, in "Algebraic Geometry" (Ann. Arbor. Mich., 1981), Lecture Notes in Math., 1008, 102-138.

[Sm] J.H. Smith, Some properties of the spectrum of a graph, in "Combinatorial Structures and Their Applications", Gordon Breach, (1970), 403-406.

[Sp] E.H. Spainer, Algebraic topology, McGraw-Hill, New York, 1966.

[Ti1] J. Tits, Groupes et géométries de Coxeter, unpublished manuscript, IHES, Paris, 1961.

[Ti2] _ Buildings of spherical type and finite BN-pairs, Lecture Notes in Mathematics, 386, Springer-Verlag, Berlin-Heidelberg-New York, 1974. 
[To] Y.L. Tong, Probability inequalities in multivariate distributions, Academic Press, New York, 1980.

[Wh] G.W. Whitehead, Elements of homotopy theory, Springer-Verlag, Berlin-HeidelbergNew York, 1978.

[Wi] E. Witt, Spiegelangsgruppen and Aufzählung halbeinfacher Liescher Ringe, Abh. Math. Sem., Hamburg, 14 (1941), 289-322.

Received June 15, 1994.

Truman State University

KiRKSVILle, Mo 63501

E-mail address: fernando@truman.edu 